\title{
Therapeutic effects of rapamycin on alcoholic cardiomyopathy
}

\author{
XILIN TU ${ }^{1 *}$, CHAO WANG ${ }^{2 *}$, XIAOXUE RU ${ }^{3}$, LILI JING ${ }^{4}$, LIJUN ZHOU $^{2}$ and LING JING ${ }^{2}$ \\ Departments of ${ }^{1}$ Emergency and ${ }^{2}$ Cardiology, The First Affiliated Hospital of Harbin Medical University; \\ ${ }^{3}$ Department of Cardiology, Harbin Second Hospital, Harbin, Heilongjiang 150001; ${ }^{4}$ Department of \\ Medicine, Rangbei Hospital, Daqing Oilfield, Daqing, Heilongjiang 163114, P.R. China
}

Received December 29, 2015; Accepted January 26, 2017

DOI: 10.3892/etm.2017.4901

\begin{abstract}
The present study aimed to investigate whether rapamycin has therapeutic potential as a treatment for alcoholic cardiomyopathy. Rats were divided into eight groups ( $n=7$ in each group): The control group; the alcohol group; abstinence in the first week; abstinence in the third week; abstinence in the fourth week; abstinent+rapamycin (AB-RAP) until the first week (AB-RAP 1); AB-RAP until the third week (AB-RAP 3); and AB-RAP until the fourth week (AB-RAP 4). Subsequently, echocardiography, and hematoxylin-eosin and Masson's staining were performed, followed by electron microscopy and terminal deoxynucleotidyl transferase-mediated dUTP nick-end labeling assay. Finally, expression levels of B cell lymphoma-2, Beclin-1 and microtubule-associated protein 1A/1B-light chain 3 were detected by immunohistochemistry and western blot analysis. The levels of left ventricular end-diastolic dimension in AB-RAP $3(7.00 \pm 0.41)$ and AB-RAP $4(6.33 \pm 0.68)$ groups were significantly lower when compared with the alcohol group $(8.01 \pm 0.30 ; \mathrm{P}<0.05)$. Compared with the alcohol group, the apoptosis rate of left ventricular myocardial tissue in the AB+RAP $3(37.68 \pm 2.15)$ and AB+RAP 4 $(26.97 \pm 2.11)$ groups was significantly reduced $(\mathrm{P}<0.05)$. To conclude, rapamycin may be considered as a therapeutic tool to attenuate alcoholic cardiomyopathy and improve cardiac function through increasing autophagy and reducing apoptosis.
\end{abstract}

\section{Introduction}

Light to moderate alcohol intake has been demonstrated to have beneficial effects on cardiovascular health $(1,2)$. However,

Correspondence to: Dr Ling Jing, Department of Cardiology, The First Affiliated Hospital of Harbin Medical University, 23 You Zheng Street, Nan Gang, Harbin, Heilongjiang 150001, P.R. China E-mail: ling_jing12@163.com

*Contributed equally

Key words: alcoholic cardiomyopathy, rapamycin, autophagy, apoptosis long-term heavy alcohol consumption, which may be considered as alcohol abuse, may cause non-ischemic dilated cardiomyopathy, referred to as alcoholic cardiomyopathy (3-5). The end-stage of alcoholic cardiomyopathy is predominantly irreversible, with evident characteristics of a significant loss of myocytes, development of progressive interstitial fibrosis and hypertrophy of the muscle cells (6). Without complete abstinence, the 4-year mortality rate of alcoholic cardiomyopathy is $\sim 50 \%$ (7).

Multiple factors are involved in the mechanisms of alcohol-induced myocardial damage, such as disruptions in protein synthesis, impaired myofibrillar structure, and disturbances in calcium transients and excitation-contraction, are implicated in the pathogenesis (8-10). Additionally, myocyte apoptosis has been shown to have essential roles in the development of structural heart damage in this disease $(11,12)$. In alcoholic patients without heart damage, anti-B cell lymphoma-2 (Bcl-2) and pro-Bcl-2-associated X protein (Bax) apoptotic mechanisms are promoted (11). Furthermore, increased expression levels of Bax and Bcl-2 are observed in the heart muscle of high-dose alcohol consumers (11).

For the treatment of patients with alcoholic heart failure, no formal guidelines have been outlined. Rapamycin, a lipophilic macrolide, was first used to suppress immune rejection in renal transplant recipients and has potential antimicrobial and antiproliferative properties (13). A previous study demonstrated that rapamycin was able to reduce cardiac fibrosis and improve cardiac function in adriamycin-induced dilated cardiomyopathy, by inhibiting the mTOR/p70s6k pathway (14). In addition, rapamycin has been indicated to have a potential role in improving age-related heart dysfunction (15). However, the exact mechanisms of rapamycin treatment on alcoholic cardiomyopathy have not been fully investigated.

In the present study, to investigate the therapeutic efficacy of rapamycin on alcoholic cardiomyopathy, a rat model of alcoholic cardiomyopathy was constructed. Echocardiography, and hematoxylin-eosin and Masson's staining were performed, followed by electron microscopy and terminal deoxynucleotidyl transferase (TdT)-mediated dUTP nick-end-labeling (TUNEL) assay. Finally, expression levels of Bcl-2, Beclin-1 and microtubule-associated protein 1A/1B-light chain 3 (LC3) were detected by immunohistochemistry and western blot analysis. 


\section{Materials and methods}

Animal model and grouping. A total of 56 male Wistar rats $(220 \pm 10 \mathrm{~g}$; 6-8 weeks old) were provided by Liaoning Changsheng Biological Technology Company (Liaoning, China). On arrival, rats were housed in a temperature-controlled vivarium $\left(22^{\circ} \mathrm{C}\right)$ with $60 \%$ humidity and 12 -h light/dark cycle. Rats had access to food and water ad libitum. Rats were randomly divided into two groups: The control group $(n=7)$ and the alcohol group $(n=49)$. Rats in the alcohol group were intragastrically administered a single dose of $60 \%$ alcohol $(6 \mathrm{ml} / \mathrm{kg} /$ day $)$ at 3:00 p.m. every day and had free access to $10 \%$ alcohol at any other time in the first week for drinking, were intragastrically administered a single dose of $60 \%$ alcohol (12 ml/kg/day) and had free access to $10 \%$ alcohol at any other time in the second week for drinking, and were intragastrically administered a single dose of $60 \%$ alcohol $(12 \mathrm{ml} / \mathrm{kg} / \mathrm{day})$ and had free access to $20 \%$ alcohol at any other time in the third week for drinking, were intragastrically administered $60 \%$ alcohol $(15 \mathrm{ml} / \mathrm{kg} /$ day, divided into two equal doses of $7.5 \mathrm{ml} / \mathrm{kg} /$ day) and had free access to $20 \%$ alcohol at any other time in weeks 4 to 16 . Rats in the control group received equivalent amounts of water. After 16 weeks, the cardiac structure and function of rats in the alcohol group were checked using an echocardiograph to verify the successful establishment of a rat alcohol model of sustained heavy alcohol intake. Subsequently, all rats in the control group and seven rats in the alcohol group were sacrificed to extract the myocardial tissue samples. To observe the role of sustained heavy alcohol intake on cardiomyopathy and the therapeutic effects of rapamycin, the other rats in the alcohol group were randomly divided into two groups ( $n=21$ per group): The abstinent (AB) group and the abstinent + rapamycin group (AB-RAP; $1.4 \mathrm{mg} / \mathrm{kg} / \mathrm{day}$ of rapamycin injected intraperitoneally). The $\mathrm{AB}$ group was again divided into three groups ( $n=7$ per group) according to the time of abstinence: Abstinence in the first week (AB 1); abstinence in the third week (AB 3); and abstinence in the fourth week (AB 4). Similarly, rats in the AB-RAP group were divided into three groups, according to the time of administration: AB-RAP in the first week (AB-RAP 1); AB-RAP in the third week (AB-RAP 3); and AB-RAP in the fourth week (AB-RAP 4).

All animal procedures conducted in the present study were approved by the Institutional Animal Use and Care Committee of the First Affiliated Hospital of Harbin Medical University (Harbin, Heilongjiang). All studies were performed according to the Principles of Laboratory Animal Care and guidelines established by the Institutional Animal Care and Use Committee of the First Affiliated Hospital of Harbin Medical University.

Echocardiography. Rats were anesthetized by intraperitoneal injection of $10 \%$ chloral hydrate $(300 \mathrm{mg} / \mathrm{kg}$; provided by the Department of Cardiology, the First Affiliated Hospital of Harbin Medical University) $24 \mathrm{~h}$ prior to procedure. Body hair was shaved in the chest region and transthoracic echocardiographic examination was performed using a $10-\mathrm{MHz}$ probe (Vivid 7; GE Healthcare Life Sciences, Chalfont, UK). Images of hearts were captured in the two-dimensional parasternal short-axis view and the levels of papillary muscles were analyzed using an M-mode echocardiogram of the mid-ventricle. Cardiac functional parameters: Left ventricular ejection fraction (LVEF), fractional shortening (FS) and left ventricular end-diastolic dimension (LVEDD) were calculated as described previously (14).

Tissue section acquisition and staining. Once rats were sacrificed, the hearts were harvested and washed with pre-cooled saline. Left ventricular myocardial tissue was dissociated out, fixed in $4 \%$ formaldehyde for $24 \mathrm{~h}$ at $22^{\circ} \mathrm{C}$ and embedded in paraffin. Sections (6- $\mu \mathrm{m}$ thick) were stained with hematoxylin and eosin and Masson's trichrome, using standard protocols for histological assessment.

Electron microscopy. Cardiac tissue samples (1-mm thick) were routinely fixed in $4 \%$ glutaraldehyde in $0.1 \mathrm{M} / 1$ phosphate-buffered saline (PBS; $\mathrm{pH} 7.2-7.4)$ for $1 \mathrm{~h}$ at $2-8^{\circ} \mathrm{C}$ and subsequently dehydrated in acetone and embedded in Epon 812. Sections were cut into 50-nm thick sections with a glass knife on an LKBi ultratome, stained with lead citrate and uranyl acetate and examined using a Hitach H-500 electron microscope (Hitachi Co., Tokyo, Japan).

TUNEL assay. TUNEL staining was performed according to the manufacturer's instructions (Roche Diagnostics, Indianapolis, IN, USA). Dewaxed and hydrated myocardial tissue paraffin slides were incubated with $1 \%$ Triton X-100 for $15 \mathrm{~min}$ at room temperature. Subsequently, the slides were incubated with $100 \mu \mathrm{l}$ TdT enzyme reaction solution (from the TUNEL staining kit) under dark and humid conditions at $37^{\circ} \mathrm{C}$ for $1 \mathrm{~h}$. A total of $100 \mu \mathrm{l}$ horseradish peroxidase-labeled streptavidin was added and incubated for $30 \mathrm{~min}$ at $37^{\circ} \mathrm{C}$, followed by two drops of freshly prepared 3,3'-diaminobenzidine solution until the reaction was terminated. Dyeing time was controlled via observation under a microscope. The slides were subsequently re-dyed, dehydrated, vitrificated, mounted and observed using a light microscope. Apoptotic cells were stained brown, while normal cells were blue-violet. The number of TUNEL-positive cells was manually counted in ten randomly selected fields of view at a magnification of $x 400$.

Immunohistochemistry and western blot analysis. For immunohistochemistry, myocardial tissue sections (6- $\mu \mathrm{m}$ thick) were deparaffinized and washed in PBS (pH 7.4) three times. After blocking with 5-10\% goat serum (Zhongshan Jinqiao Biotechnology Co., Ltd., Beijing, China) for $30 \mathrm{~min}$ at $37^{\circ} \mathrm{C}$, slides were incubated with anti-Beclin-1 rabbit polyclonal antibody $(1: 1,000)$, anti-LC3 rabbit polyclonal antibody $(1: 1,000)$ and anti-Bcl-2 rabbit polyclonal antibody (1:200; all Santa Cruz Biotechnology, Santa Cruz, CA, USA) at $4^{\circ} \mathrm{C}$ overnight, followed by incubation with biotinylated secondary antibody (1:1,000; Zhongshan Jinqiao Biotechnology Co., Ltd.) at room temperature for 10-30 min. Immunohistochemical staining followed by diaminobenzidine staining was subsequently performed. All sections were counterstained with hematoxylin and dehydrated, cleared, mounted and viewed under a light microscope at magnification, x200.

Tissue samples from the left ventricle were homogenized and the protein was extracted using an animal and plant protein extraction kit (Beyotime Institute of Biotechnology, Haimen, 
China). The total protein concentration was determined using the bicinchoninic acid assay method. Following this, $50 \mu \mathrm{g}$ protein sample was separated using 10\% SDS-PAGE and transferred onto nitrocellulose membrane. The membranes were blocked at room temperature for $1 \mathrm{~h}$ and incubated at $4^{\circ} \mathrm{C}$ overnight with one of the following antibodies: Anti-Beclin-1 rabbit polyclonal antibody $(1: 1,000)$, anti-LC3 rabbit polyclonal antibody $(1: 1,000)$, anti-Bcl-2 rabbit polyclonal antibody (1:200) and $\beta$-actin (1:1,000; loading control; all Zhongshan Jinqiao Biotechnology Co., Ltd.). Subsequently, membranes were rinsed three times in tris-buffered saline-Tween-20, for $10 \mathrm{~min}$ each, and incubated in secondary alkaline-phosphatase-conjugated goat anti-rabbit immunoglobulin G (1:5,000; Santa Cruz Biotechnology) at $4^{\circ} \mathrm{C}$ for $90 \mathrm{~min}$. Following washing, the relative intensity of the bands produced from the immunoblots was measured using enhanced chemiluminescence based on a gel documentation analysis system (Bio-Rad Laboratories, Inc., Hercules, CA, USA).

Statistical analysis. Data were expressed as mean \pm standard deviation unless otherwise stated. Results were analyzed by one way analysis of variance and Student's t-test was performed. For comparing the mean among multiple samples, the q-test was used. All analyses were performed with SPSS 18.0 software (SPSS, Inc., Chicago, IL, USA). P<0.05 was considered to indicate a statistically significant difference.

\section{Results}

Echocardiography. Echocardiographic data in all groups are indicated in Table I. The level of LVEDD in the alcohol group was significantly increased when compared with the control group (8.01 \pm 0.30 vs. 5.29 \pm 0.57 ; $\mathrm{P}<0.05)$. LVEF and FS were significantly decreased in the alcohol group when compared with the control group $(\mathrm{P}<0.05)$. The levels of LVEDD in

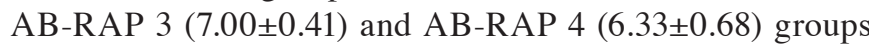
were significantly decreased when compared with the alcohol group $(8.01 \pm 0.30 ; \mathrm{P}<0.05)$. LVEF and FS in AB-RAP 3 and AB-RAP 4 groups were significantly increased when compared with the alcohol group $(\mathrm{P}<0.05)$. Additionally, when compared with the AB-RAP 3 group, the level of LVEDD was significantly decreased $(\mathrm{P}<0.05)$ and the LVEF and FS were markedly higher in the AB-RAP 4 group $(\mathrm{P}>0.05)$. No significant differences in the levels of LVEDD, LVEF and FS were observed between the AB-RAP1, AB 1, AB 3 or $\mathrm{AB} 4$ group when compared with the alcohol group $(\mathrm{P}>0.05)$, respectively.

Hematoxylin and eosin and Masson's staining. To evaluate the effects of rapamycin on alcoholic cardiomyopathy, histopathological changes were investigated via hematoxylin and eosin staining (Fig. 1) and Masson's staining (Fig. 2). As demonstrated in Fig. 1, in the control group, cardiac muscle fibers were arranged in order, the cytoplasm was uniform and no cellular infiltration was indicated. Cardiac muscle fiber arrangement disorder, a large number of cell necrosis and cellular infiltration was observed in the alcohol group, and the same was demonstrated in the AB groups. However, in the AB-RAP 1, AB-RAP 3 and AB-RAP 4 groups, less cell necrosis and cellular infiltration was observed and the cardiac
Table I. Echocardiographic parameters.

\begin{tabular}{lcll}
\hline Group (n=7) & LVEDD (mm) & LVEF (\%) & \multicolumn{1}{c}{ FS (\%) } \\
\hline Control & $5.29 \pm 0.57$ & $76.60 \pm 2.07$ & $49.40 \pm 3.36$ \\
Alcohol & $8.01 \pm 0.30^{\mathrm{a}}$ & $45.20 \pm 1.92^{\mathrm{a}}$ & $30.29 \pm 2.74^{\mathrm{a}}$ \\
AB 1 & $8.03 \pm 0.29$ & $46.20 \pm 1.09$ & $30.80 \pm 3.11$ \\
AB-RAP 1 & $7.94 \pm 0.29$ & $45.40 \pm 3.05$ & $31.40 \pm 2.88$ \\
AB 3 & $7.97 \pm 0.36$ & $47.60 \pm 1.95$ & $31.00 \pm 2.92$ \\
AB-RAP 3 & $7.00 \pm 0.41^{\mathrm{b}}$ & $52.40 \pm 2.76^{\mathrm{b}}$ & $34.60 \pm 2.07^{\mathrm{b}}$ \\
AB 4 & $7.59 \pm 0.31$ & $49.80 \pm 4.97$ & $32.40 \pm 3.21$ \\
AB-RAP 4 & $6.33 \pm 0.68^{\mathrm{b}, \mathrm{c}}$ & $56.20 \pm 2.39^{\mathrm{b}, \mathrm{c}}$ & $37.80 \pm 1.92^{\mathrm{b}, \mathrm{c}}$
\end{tabular}

${ }^{\mathrm{a}} \mathrm{P}<0.05$ vs. control group; ${ }^{\mathrm{b}} \mathrm{P}<0.05$ vs. alcohol group; ${ }^{\mathrm{c}} \mathrm{P}<0.05$ vs. AB-RAP 3. LVEDD, left ventricle end-diastolic dimension; LVEF, left ventricle ejection fraction; FS, fractional shortening. AB 1 group, abstinent in the first week; $\mathrm{AB} 3$ group, abstinent in the third week; AB 4 group, abstinent in the fourth week; AB-RAP 1 group, abstinentin the first week + rapamycin $(1.4 \mathrm{mg} / \mathrm{kg} / \mathrm{day}) ; \mathrm{AB}-\mathrm{RAP} 3$, abstinent in the third week + rapamycin $(1.4 \mathrm{mg} / \mathrm{kg} /$ day $)$; AB-RAP 4 , abstinent in the fourth week + rapamycin $(1.4 \mathrm{mg} / \mathrm{kg} /$ day $)$; Alcohol group, varying concentrations of $60 \%$ alcohol over the period of four weeks; Control group, rats received similar quantities of water as the alcohol group.

muscle fiber was compactly arranged. Compared with the control group, increased myocardial fibrosis was observed in the alcohol group (Fig. 2). The extent of myocardial fibrosis in the AB group was consistent with the alcohol group. Additionally, myocardial fibrosis was reduced in the AB-RAP group when compared with the alcohol group.

Electron microscopy. Cellular structure, such as the nucleus, mitochondria, and the cytoplasm of the muscle cells, were indicated to be normal in the control group (Fig. 3). Muscle fibers were arranged closely without fracture. However, in the alcohol group, the nucleus was swollen and dissolved, the muscle plasma was expansive, the mitochondria were also swollen and the autophagy bodies were visible, which was also observed with the AB group. The cell structure damage in all $\mathrm{AB}+\mathrm{RAP}$ groups appeared to be relieved when compared with the alcohol group.

Analysis of apoptosis. Apoptotic left ventricular myocardial tissue was stained brown (Fig. 4). The apoptotic rate of left ventricular myocardial tissue was significantly increased in the alcohol group $(61.38 \pm 3.98)$ when compared with the control group $(20.48 \pm 2.32 ; \mathrm{P}<0.05$; Table II). No significant difference in the rate of apoptosis was observed between the alcohol group and the AB + RAP 1 group $(\mathrm{P}>0.05)$. However, compared with the alcohol group, the apoptosis rate of left ventricular myocardial tissue in the AB+RAP $3(37.68 \pm 2.15)$ and $\mathrm{AB}+\mathrm{RAP} 4(26.97 \pm 2.11)$ groups was significantly decreased $(\mathrm{P}<0.05)$.

Expression of Bcl-2, Beclin 1 and LC3. Following analysis of the protein expression levels of Bcl-2, Beclin-1 and LC3 by western blot, it was apparent that expression occurred in every group (Fig. 5A). The relative intensities of Beclin-1 
A

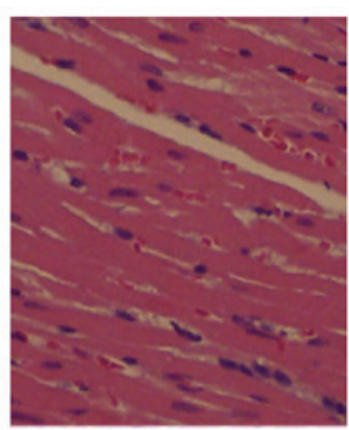

$\mathbf{E}$

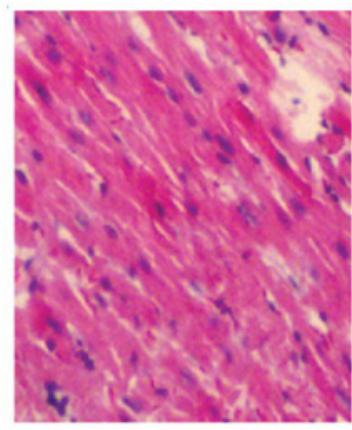

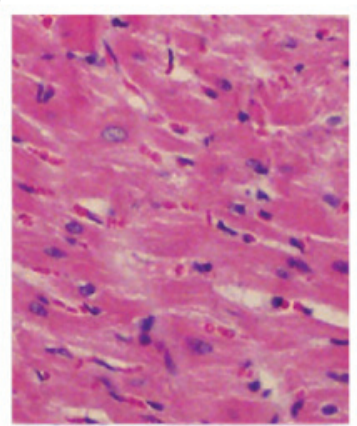

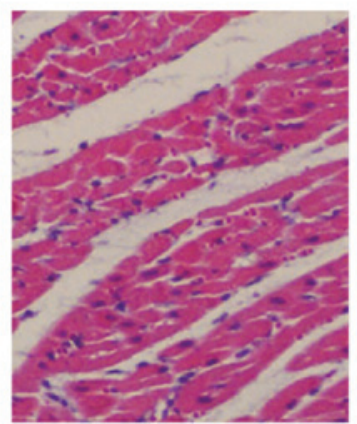

C
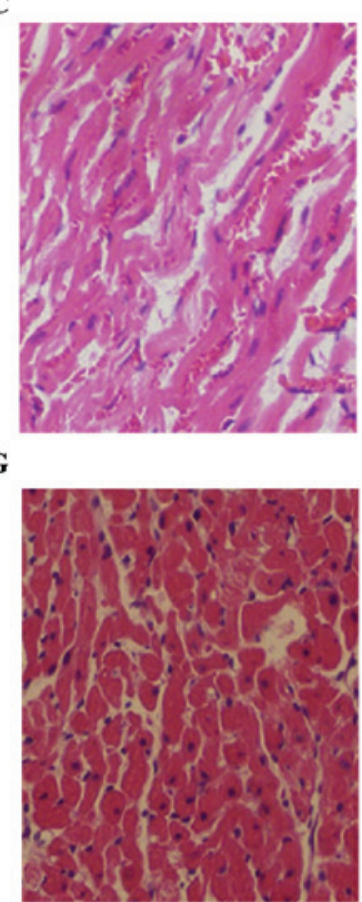

D

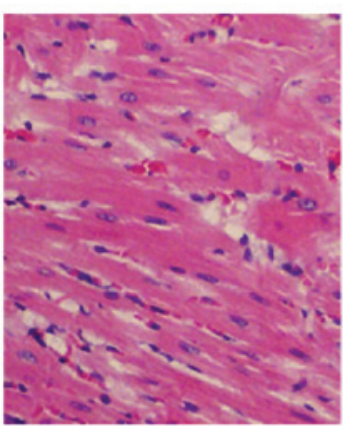

$\mathbf{H}$

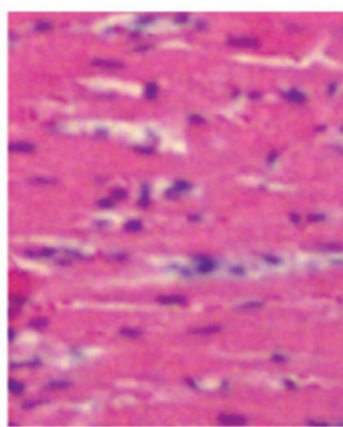

Figure 1. Effects of rapamycin on cardiomyopathy detected by hematoxylin and eosin staining (magnification, x200). (A) Control group rats received similar quantities of water as the alcohol group; (B) Alcohol group rats received varying concentrations of $60 \%$ alcohol over the period of four weeks; (C) AB 1 group rats were $\mathrm{AB}$ in the first week; (D) $\mathrm{AB}-\mathrm{RAP} 1$ group rats were $\mathrm{AB}$ in the first week and received $\mathrm{RAP}(1.4 \mathrm{mg} / \mathrm{kg} / \mathrm{day}) ;(\mathrm{E}) \mathrm{AB} 3 \mathrm{group}$ rats were $\mathrm{AB}$ in the third week; (F) AB-RAP 3 rats were AB in the third week and received RAP (1.4 mg/kg/day); (G) AB 4 group rats were AB in the fourth week; and (H) AB-RAP 4 rats were $\mathrm{AB}$ in the fourth week and received RAP (1.4 mg/kg/day). AB, abstinent; RAP, rapamycin.

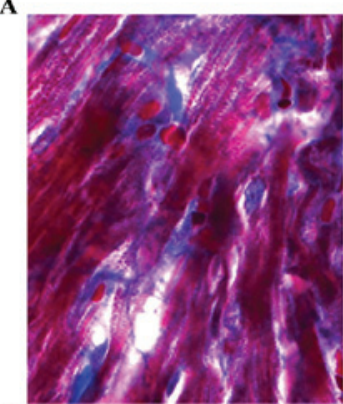

$\mathbf{E}$

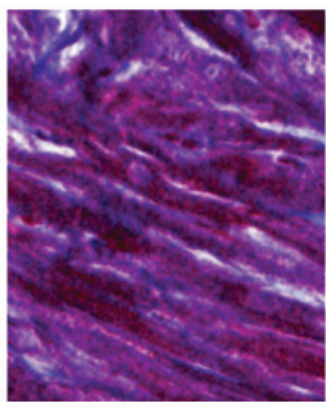

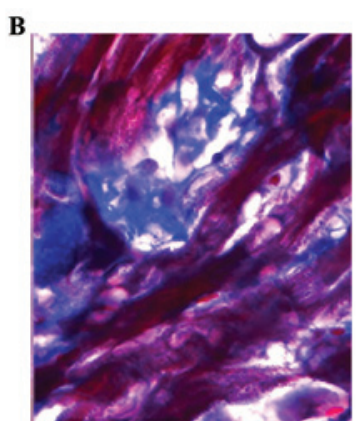

F

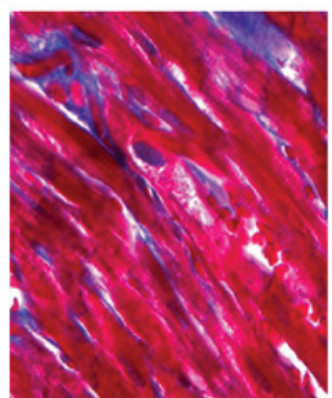

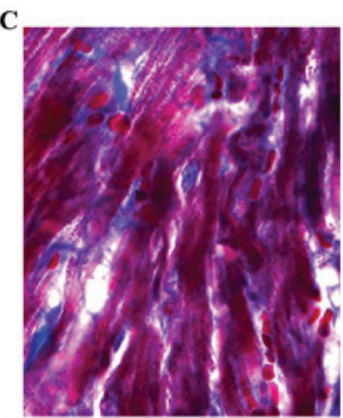

G

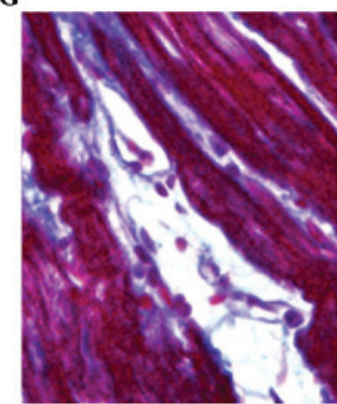

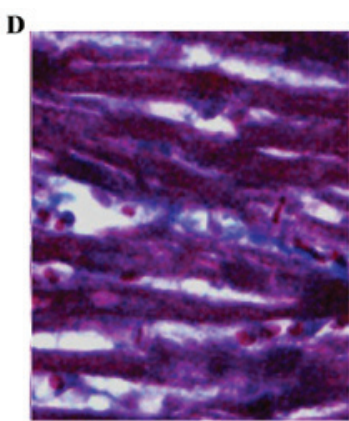

H

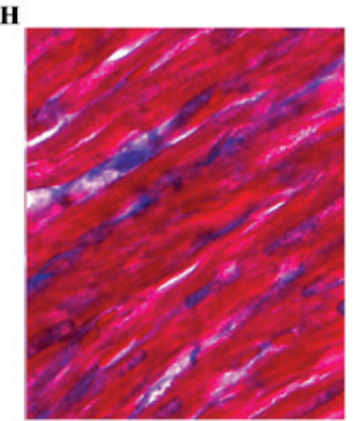

Figure 2. Effects of rapamycin on cardiomyopathy detected by Masson's staining (magnification, x400). (A) Control group rats received similar quantities of water as the alcohol group; (B) Alcohol group rats received varying concentrations of $60 \%$ alcohol over the period of four weeks; (C) AB 1 group rats were $\mathrm{AB}$ in the first week; (D) AB-RAP 1 group rats were AB in the first week and received RAP (1.4 mg/kg/day); (E) AB 3 group rats were AB in the third week; (F) AB-RAP 3 rats were AB in the third week and received RAP (1.4 mg/kg/day); $(\mathrm{G}) \mathrm{AB} 4$ group rats were AB in the fourth week; and (H) AB-RAP 4 rats were $\mathrm{AB}$ in the fourth week and received RAP $(1.4 \mathrm{mg} / \mathrm{kg} /$ day $) . \mathrm{AB}$, abstinent; RAP, rapamycin.

and LC3 bands were significantly higher, whereas the relative intensity of the $\mathrm{Bcl}-2$ were lower, in the alcohol group when compared with the control group (Fig. 5B; $\mathrm{P}<0.05$ ).
The relative intensities of Bcl-2, Beclin-1 and LC3 bands were higher in the AB-RAP 3 and AB-RAP 4 group when compared with the alcohol group $(\mathrm{P}<0.05)$. No significant 
$\mathbf{A}$

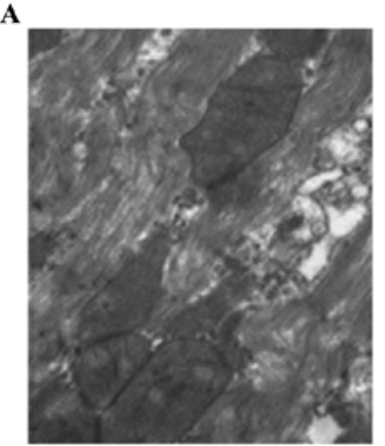

$\mathbf{E}$

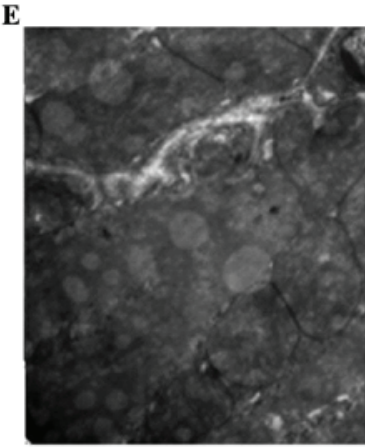

B

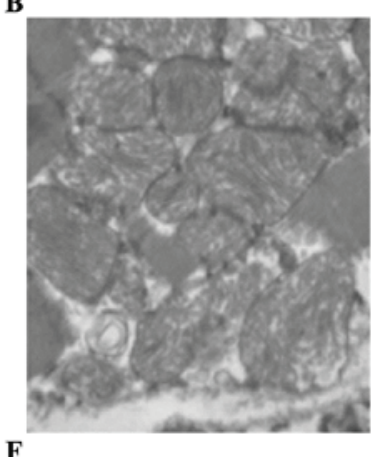

F

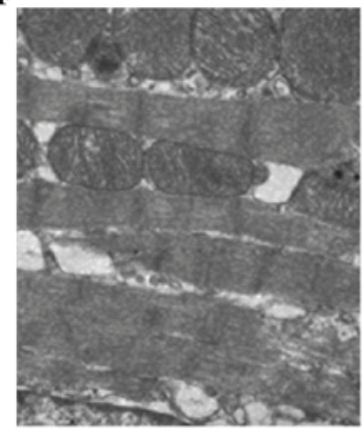

C

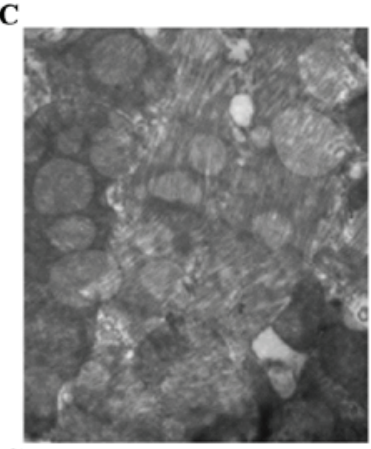

G

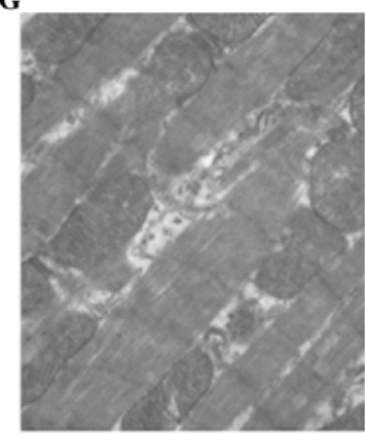

D

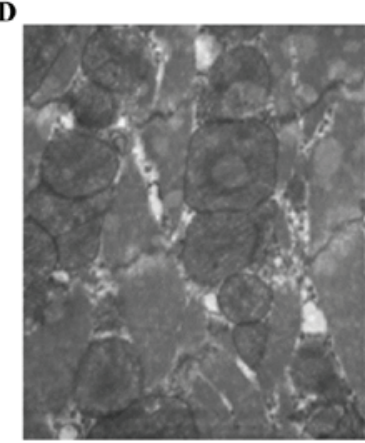

H

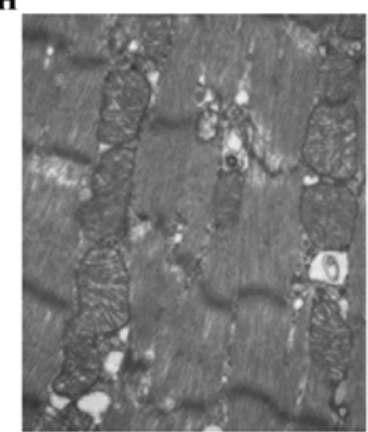

Figure 3. Effects of rapamycin on cardiomyopathy detected by electron microscopy (magnification, x20,000). (A) Control group rats received similar quantities of water as the alcohol group; (B) Alcohol group rats received varying concentrations of $60 \%$ alcohol over the period of four weeks; (C) AB 1 group rats were $\mathrm{AB}$ in the first week; (D) AB-RAP 1 group rats were AB in the first week and received $\mathrm{RAP}(1.4 \mathrm{mg} / \mathrm{kg} / \mathrm{day})$; (E) AB 3 group rats were AB in the third week; (F) AB-RAP 3 rats were AB in the third week and received RAP (1.4 mg/kg/day); (G) AB 4 group rats were AB in the fourth week; and (H) AB-RAP 4 rats were $\mathrm{AB}$ in the fourth week and received RAP $(1.4 \mathrm{mg} / \mathrm{kg} /$ day $) . \mathrm{AB}$, abstinent; RAP, rapamycin.

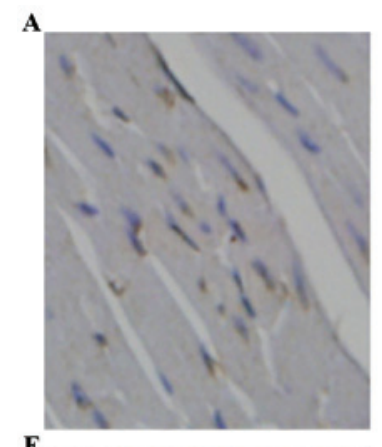

$\mathbf{E}$

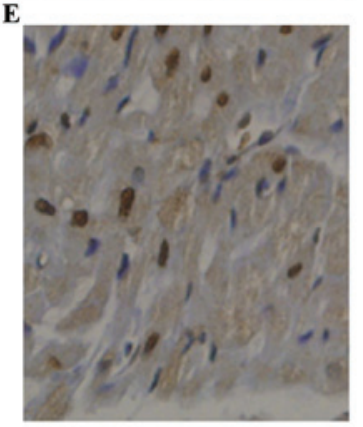

B

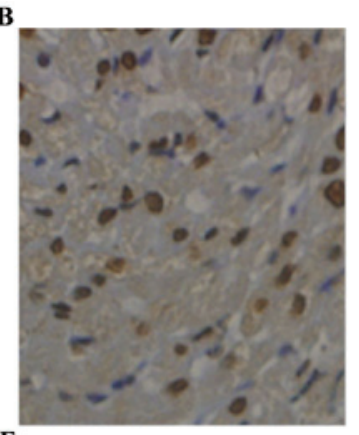

F

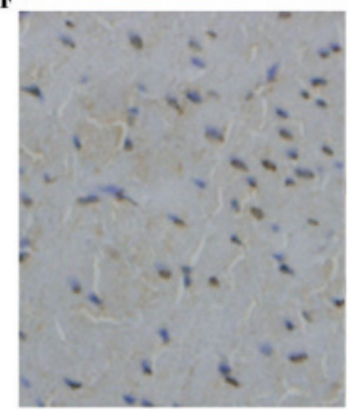

c

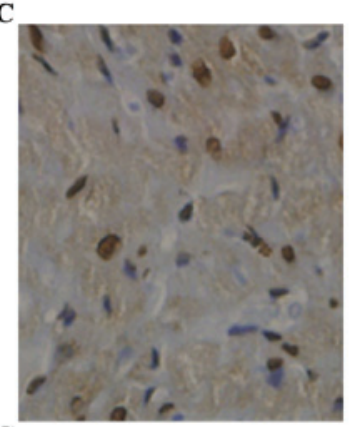

G

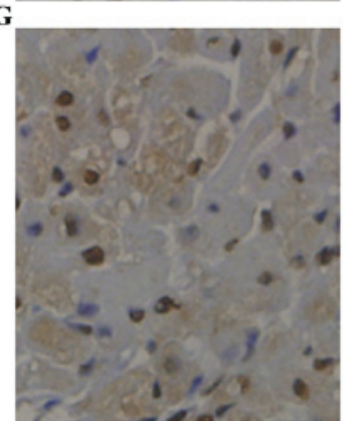

D

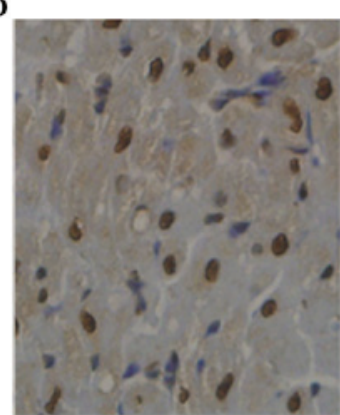

H

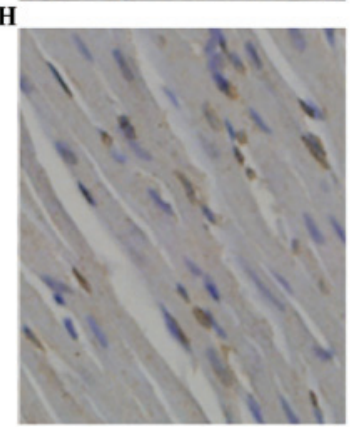

Figure 4. Level of apoptosis in each group (magnification, x400). (A) Control group rats received similar quantities of water as the alcohol group; (B) Alcohol group rats received varying concentrations of $60 \%$ alcohol over the period of four weeks; (C) AB 1 group rats were AB in the first week; (D) AB-RAP 1 group rats were $\mathrm{AB}$ in the first week and received $\mathrm{RAP}(1.4 \mathrm{mg} / \mathrm{kg} / \mathrm{day})$; (E) $\mathrm{AB} 3$ group rats were $\mathrm{AB}$ in the third week; (F) $\mathrm{AB}-\mathrm{RAP} 3 \mathrm{rats}$ were $\mathrm{AB}$ in the third week and received RAP (1.4 mg/kg/day); (G) AB 4 group rats were AB in the fourth week; and (H) AB-RAP 4 rats were AB in the fourth week and received RAP (1.4 mg/kg/day). AB, abstinent; RAP, rapamycin.

difference of relative intensities of Bcl-2, Beclin-1 and LC3 bands were observed between the alcohol group and $A B$ 1, AB 3 or AB-RAP 1 groups $(\mathrm{P}>0.05)$.
Immunohistochemical analysis of Bcl-2, Beclin-1 and LC3 expression levels demonstrated the presence of all proteins in the samples. In agreement with the western 


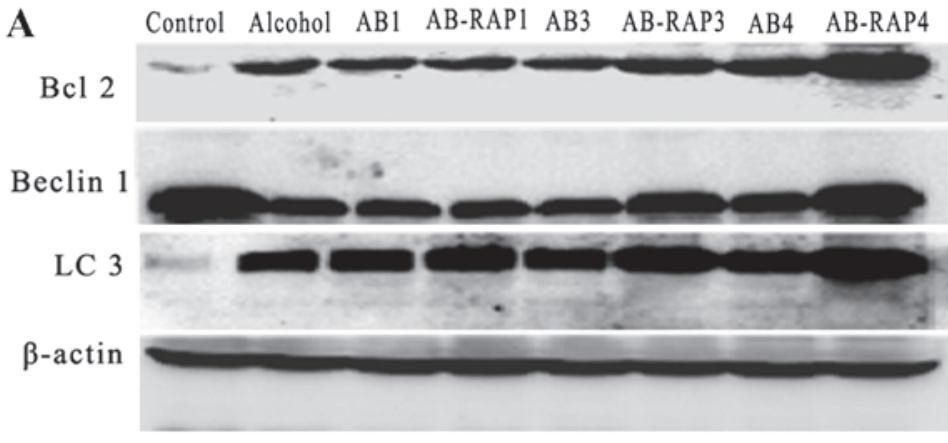

B

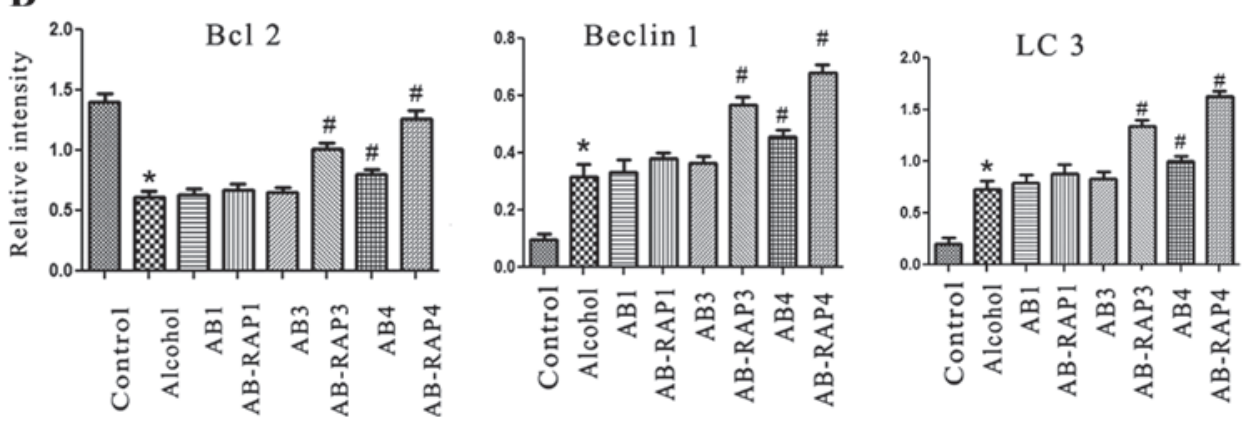

Figure 5. (A) Protein expression levels and (B) relative band intensities of Bcl-2, Beclin-1 and LC3 in each group. Control group rats received similar quantities of water as the alcohol group. Alcohol group rats received varying concentrations of $60 \%$ alcohol over the period of four weeks. AB 1 group rats were $\mathrm{AB}$ in the first week. AB-RAP 1 group rats were $A B$ in the first week and received $\mathrm{RAP}(1.4 \mathrm{mg} / \mathrm{kg} / \mathrm{day})$. AB 3 group rats were $\mathrm{AB}$ in the third week. $\mathrm{AB}-\mathrm{RAP} 3$ rats were $\mathrm{AB}$ in the third week and received RAP $(1.4 \mathrm{mg} / \mathrm{kg} /$ day $)$. AB 4 group rats were $\mathrm{AB}$ in the fourth week. AB-RAP 4 rats were $\mathrm{AB}$ in the fourth week and received RAP $(1.4 \mathrm{mg} / \mathrm{kg} /$ day $)$. Data are presented as the mean \pm standard deviation. ${ }^{*} \mathrm{P}<0.05$ vs. the control group; ${ }^{*} \mathrm{P}<0.05$ vs. the alcohol group. $\mathrm{AB}$, abstinent; RAP, rapamycin; Bcl-2, B cell lymphoma-2; LC3, microtubule-associated protein 1A/B-light chain 3.

Table II. Apoptotic rate in each group.

\begin{tabular}{lc}
\hline Group $(\mathrm{n}=7)$ & Apoptotic rate $(\%)$ \\
\hline Control & $20.48 \pm 2.32$ \\
Alcohol & $61.38 \pm 3.98^{\mathrm{a}}$ \\
AB 1 & $60.06 \pm 5.66$ \\
AB-RAP 1 & $58.38 \pm 4.61$ \\
AB 3 & $58.21 \pm 6.29$ \\
AB-RAP 3 & $37.68 \pm 2.15^{\mathrm{b}}$ \\
AB 4 & $52.56 \pm 2.98^{\mathrm{b}}$ \\
AB-RAP 4 & $26.97 \pm 2.11^{\mathrm{b}}$ \\
\hline
\end{tabular}

Data are presented as the mean \pm standard deviation. ${ }^{\mathrm{a}} \mathrm{P}<0.05$ vs. control group; ${ }^{\mathrm{b}} \mathrm{P}<0.05$ vs. alcohol group. $\mathrm{AB} 1$ group, abstinent in the first week; AB 3 group, abstinent in the third week; AB 4 group, abstinent in the fourth week; AB-RAP 1 group, abstinent in the first week + rapamycin $(1.4 \mathrm{mg} / \mathrm{kg} / \mathrm{day}) ; \mathrm{AB}-\mathrm{RAP} 3$, abstinent in the third week + rapamycin $(1.4 \mathrm{mg} / \mathrm{kg} /$ day $)$; AB-RAP 4 , abstinent in the fourth week + rapamycin $(1.4 \mathrm{mg} / \mathrm{kg} / \mathrm{day})$; Alcohol group, varying concentrations of $60 \%$ alcohol over the period of four weeks; Control group, rats received similar quantities of water as the alcohol group.

blot analysis, the sum integrated optical density (IOD) of Beclin-1 and LC3 was significantly increased, whereas the sum IOD of Bcl-2 was significantly decreased, in the alcohol group when compared with the control group (Fig. 6; $\mathrm{P}<0.05)$. Furthermore, the sum IOD of Bcl-2, Beclin-1 and LC3 expression levels were significantly increased in the
AB-RAP 3 and AB-RAP 4 groups when compared with the alcohol group $(\mathrm{P}<0.05)$.

\section{Discussion}

The present study investigated the treatment of rapamycin on alcoholic cardiomyopathy. Alcoholic cardiomyopathy is characterized by left ventricular dilatation, normal or decreased left ventricular wall thickness, increased left ventricular mass and decreased left ventricular ejection fraction $(<40 \%)(16)$. Autophagy and apoptosis are two predominant processes of cell death, which have important roles in the pathogenesis of alcoholic cardiomyopathy (12). In the present study, to investigate the role of rapamycin on alcoholic cardiomyopathy, changes of autophagy and apoptosis in rats with alcoholic cardiomyopathy were observed.

Dead cardiomyocytes indicating autophagy traits have been previously reported in heart failure caused by dilated cardiomyopathy (17). The mammalian target of rapamycin (mTOR) is a type of phosphatidylinositol kinase that is able to negatively regulate autophagy (18). Rapamycin, a lipophilic macrolide antibiotic, is able to induce autophagy through the inactivation of mTOR (19). Alternative studies have claimed that the mTOR has beneficial effects towards cardiac function in alcoholic cardiomyopathy $(20,21)$. Therefore, autophagy may be negatively regulated by the mTOR and Ras-cAMP-protein kinase A (PKA) pathways.

LC3 and Beclin-1 are important proteins associated with autophagy (22).LC3 is mediated by PKA and its phosphorylation 

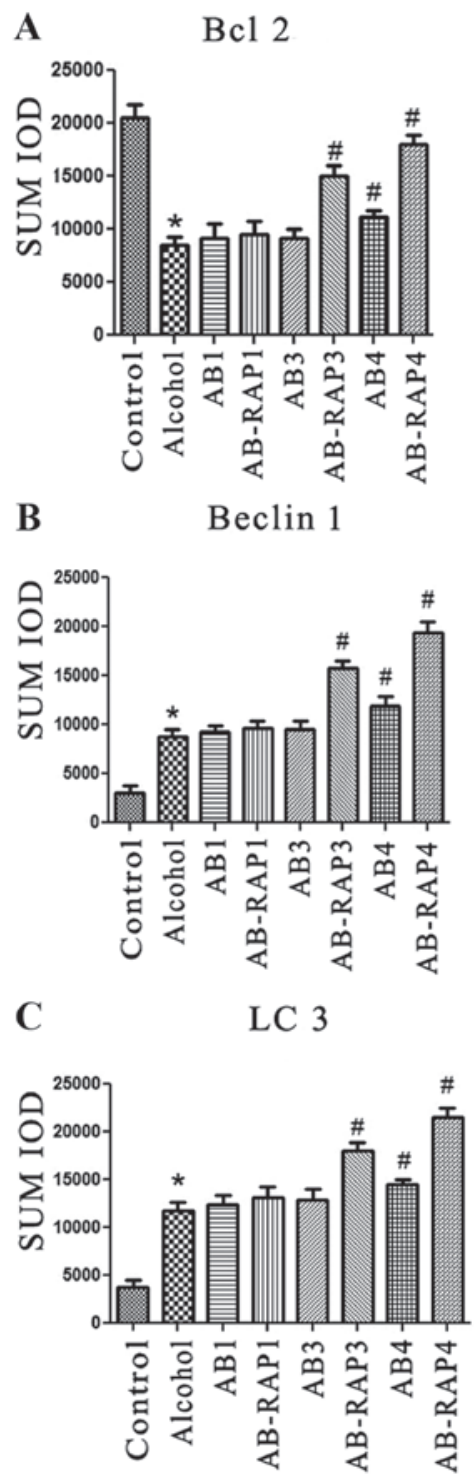

Figure 6. Sum IOD levels of (A) Bcl-2, (B) Beclin 1 and (C) LC3 expression in each group. Data are presented as the mean \pm standard deviation. $\mathrm{AB} 1$ group, abstinent in the first week; $\mathrm{AB} 3$ group, abstinent in the third week; AB 4 group, abstinent in the fourth week; AB-RAP 1 group, abstinen in the first week + rapamycin $(1.4 \mathrm{mg} / \mathrm{kg} /$ day $) ; \mathrm{AB}-\mathrm{RAP} 3$, abstinent in the third week + rapamycin $(1.4 \mathrm{mg} / \mathrm{kg} /$ day $) ; \mathrm{AB}-\mathrm{RAP} 4$, abstinent in the fourth week + rapamycin $(1.4 \mathrm{mg} / \mathrm{kg} / \mathrm{day})$; Alcohol group, varying concentrations of $60 \%$ alcohol over the period of four weeks; Control group, rats received similar quantities of water as the alcohol group." $\mathrm{P}<0.05$ vs. the control group; ${ }^{\text {"}} \mathrm{P}<0.05$ vs. the alcohol group. Bcl-2, B cell lymphoma-2; LC3, microtubule-associated protein 1A/B-light chain 3; IOD, integrated optical density.

prevents LC3 recruitment to autophagosomes (23). Beclin-1 is a newly discovered phosphorylation substrate linked to death associated protein kinase (DAPK) (24). DAPK is able to stimulate the functions of cell death and growth and promote autophagy and membrane blebbing through binding to LC3 (25). Furthermore, DAPK phosphorylates Beclin-1 and is able to trigger autophagy by promoting the separation of Beclin-1 from Bcl-2-like proteins (24). In the present study, after analyzing the expression levels of beclin-1 and LC3, the relative intensities and the sum IOD of Beclin- 1 and LC3 were indicated to be significantly higher in the AB-RAP 3 and AB-RAP 4 groups when compared with the alcohol group, which suggests that rapamycin may improve cardiac function in rats with alcoholic cardiomyopathy, through increasing autophagy.

Cardiac apoptosis is a fundamental indicator of various cardiomyopathies (12). Previous studies have verified that apoptosis increases in the heart of animals and patients with chronic alcohol consumption $(11,26)$. In a similar manner, the present study demonstrated that the apoptosis rate of left ventricular myocardial tissue was significantly increased in the alcohol group when compared with the control group. Bcl-2 functions as a dominant regulator of apoptosis that is able to inhibit most types of apoptotic cell death (27). A previous study has shown that rapamycin is able to increase the cellular concentration of the Bcl-2 protein and thereby exert its anti-apoptotic effect (28). Additionally, an alternative study revealed that rapamycin inhibits the function of mTOR by combining with FKBP12, suppresses the function of its downstream-related factors and block cells in G1 phase, thereby inducing apoptosis (29). In the present study, the relative intensities and the sum IOD of Bcl-2 were indicated to be significantly higher in the AB-RAP 3 and AB-RAP 4 groups when compared with the alcohol group. To conclude, this indicates that rapamycin may improve cardiac function in rats with alcoholic cardiomyopathy, through reducing apoptosis.

The present study exhibited certain limitations. Research concerning the role of rapamycin on autophagy was performed through analyzing the expression levels of LC3 and Beclin-1; however, the pathway of autophagy was not specifically studied. Additionally, a singular concentration of rapamycin was analyzed and the relationship between the dose and effect was not evaluated. Furthermore, to determine the effects that rapamycin alone has on cardiac function, a control group with rapamycin in absence of alcohol should have been indicated. Moreover, experiments were terminated at 4 weeks post-abstinence and therefore did not consider results past this time-frame. Thus, further studies on the effect of rapamycin on alcoholic cardiomyopathy are required.

In conclusion, the present study indicates that rapamycin may be a useful therapeutic tool to attenuate alcoholic cardiomyopathy and improve cardiac function, by increasing autophagy and reducing apoptosis.

\section{Acknowledgements}

The present study was supported by The National Natural Science Foundation of China (grant no. 81570341) and Heilongjiang Province Postdoctoral Science Foundation (grant no. LBH-Q12050).

\section{References}

1. Bryson CL, Mukamal KJ, Mittleman MA, Fried LP, Hirsch CH, Kitzman DW and Siscovick DS: The association of alcohol consumption and incident heart failure: The cardiovascular health study. J Am Coll Cardiol 48: 305-311, 2006.

2. Movva R and Figueredo VM: Alcohol and the heart: To abstain or not to abstain? Int J Cardiol 164: 267-276, 2013.

3. Guzzo-Merello G, Cobo-Marcos M, Gallego-Delgado M and Garcia-Pavia P: Alcoholic cardiomyopathy. World J Cardiol 6: 771-781, 2014.

4. Gavazzi A, De Maria R, Parolini M and Porcu M: Alcohol abuse and dilated cardiomyopathy in men. Am J Cardiol 85: 1114-1118, 2000 . 
5. Fauchier L, Babuty D, Poret P, Casset-Senon D, Autret ML, Cosnay P and Fauchier JP: Comparison of long-term outcome of alcoholic and idiopathic dilated cardiomyopathy. Eur Heart J 21: 306-314, 2000

6. Fernandez-Sola J, Estruch R, Grau JM, Pare JC, Rubin E and Urbano-Marquez A: The relation of alcoholic myopathy to cardiomyopathy. Ann Intern Med 120: 529-536, 1994.

7. Laonigro I, Correale M, Di Biase M and Altomare E: Alcohol abuse and heart failure. Eur J Heart Fail 11: 453-462, 2009.

8. Molina PE, McClain C, Valla D, Guidot D, Diehl AM, Lang CH and Neuman M: Molecular pathology and clinical aspects of alcohol-induced tissue injury. Alcohol Clin Exp Res 26: 120-128, 2002.

9. Ren J and Wold LE: Mechanisms of alcoholic heart disease. Ther Adv Cardiovasc Dis 2: 497-506, 2008.

10. Urbano-Márquez A and Fernández-Solà J: The effect of alcohol on cardiac and skeletal muscle. Muscle Nerve 30: 689-707, 2004.

11. Fernández-Solà J, Fatjó F, Sacanella E, Estruch R, Bosch X, Urbano-Márquez A and Nicolás JM: Evidence of apoptosis in alcoholic cardiomyopathy. Human Pathol 37: 1100-1110, 2006.

12. Gürtl B, Kratky D, Guelly C, Zhang L, Gorkiewicz G, Das SK, Tamilarasan KP and Hoefler G: Apoptosis and fibrosis are early features of heart failure in an animal model of metabolic cardiomyopathy. Int J Exp Pathol 90: 338-346, 2009.

13. Saunders RN, Metcalfe MS and Nicholson ML: Rapamycin in transplantation: A review of the evidence. Kidney Int 59: 3-16, 2001.

14. Yu SY,Liu L,LiP and Li J: Rapamycin inhibits the mTOR/p70S6K pathway and attenuates cardiac fibrosis in adriamycin-induced dilated cardiomyopathy. Thorac Cardiovasc Surg 61: 223-228, 2013.

15. Flynn JM, O'Leary MN, Zambataro CA, Academia EC, Presley MP, Garrett BJ, Zykovich A, Mooney SD, Strong R, Rosen CJ, et al: Late-life rapamycin treatment reverses age-related heart dysfunction. Aging Cell 12: 851-862, 2013.

16. Piano MR and Phillips SA: Alcoholic cardiomyopathy: Pathophysiologic insights. Cardiovasc Toxicol 14: 291-308, 2014.

17. Shimomura H, Terasaki F, Hayashi T, Kitaura Y, Isomura $T$ and Suma H: Autophagic degeneration as a possible mechanism of myocardial cell death in dilated cardiomyopathy. Jpn Circ J 65: 965-968, 2001.

18. Kim J, Kundu M, Viollet B and Guan KL: AMPK and mTOR regulate autophagy through direct phosphorylation of Ulk1. Nat Cell Biol 13: 132-141, 2011.
19. Ravikumar B, Vacher C, Berger Z, Davies JE, Luo S, Oroz LG, Scaravilli F, Easton DF, Duden R, O'Kane CJ and Rubinsztein DC: Inhibition of mTOR induces autophagy and reduces toxicity of polyglutamine expansions in fly and mouse models of Huntington disease. Nat Genet 36: 585-595, 2004.

20. Li Q and Ren J: Cardiac overexpression of metallothionein rescues chronic alcohol intake-induced cardiomyocyte dysfunction: Role of Akt, mammalian target of rapamycin and ribosomal p70s6 kinase. Alcohol Alcohol 41: 585-592, 2006.

21. Ge W and Ren J: mTOR-STAT3-notch signalling contributes to ALDH2-induced protection against cardiac contractile dysfunction and autophagy under alcoholism. J Cell Mol Med 16: 615-625, 2012

22. Oh SY, Choi SJ, Kim KH, Cho EY, Kim JH and Roh CR: Autophagy-related proteins, LC 3 and Beclin-1, in placentas from pregnancies complicated by preeclampsia. Reprod Sci 15 : 912-920, 2008.

23. Cherra SJ III, Kulich SM, Uechi G, Balasubramani M, Mountzouris J, Day BW and Chu CT: Regulation of the autophagy protein LC3 by phosphorylation. J Cell Biol 190: 533-539, 2010.

24. Zalckvar E, Berissi H, Mizrachy L, Idelchuk Y, Koren I, Eisenstein M, Sabanay H, Pinkas-Kramarski R and Kimchi A: DAP-kinase-mediated phosphorylation on the $\mathrm{BH} 3$ domain of beclin 1 promotes dissociation of beclin 1 from Bcl-XL and induction of autophagy. EMBO Rep 10: 285-292, 2009.

25. Gandesiri M, Chakilam S, Ivanovska J, Benderska N, Ocker M, Di Fazio P, Feoktistova M, Gali-Muhtasib H, Rave-Fränk M, Prant $\mathrm{O}$, et al: Erratum to: DAPK plays an important role in panobinostat-induced autophagy and commits cells to apoptosis under autophagy deficient conditions. Apoptosis 21: 671-674, 2016.

26. Capasso JM, Li P, Guideri G, Malhotra A, Cortese R and Anversa P: Myocardial mechanical, biochemical, and structural alterations induced by chronic ethanol ingestion in rats. Circ Res 71: 346-356, 1992.

27. Hockenbery DM, Oltvai ZN, Yin XM, Milliman CL and Korsmeyer SJ: Bcl-2 functions in an antioxidant pathway to prevent apoptosis. Cell 75: 241-251, 1993.

28. Calastretti A, Rancati F, Ceriani MC, Asnaghi L, Canti G and Nicolin A: Rapamycin increases the cellular concentration of the BCL-2 protein and exerts an anti-apoptotic effect. Eur J Cancer 37: 2121-2128, 2001.

29. Ravikumar B,BergerZ, VacherC, O'Kane CJ and Rubinsztein DC: Rapamycin pre-treatment protects against apoptosis. Hum Mol Genet 15: 1209-1216, 2006 